\title{
Adrenal hyperandrogenism does not deteriorate insulin resistance and lipid profile in women with PCOS
}

\author{
Stavroula A Paschou', Eleni Palioura', Dimitrios loannidis², \\ Panagiotis Anagnostis ${ }^{3}$, Argyro Panagiotakou'², Vasiliki Loi' \\ Georgios Karageorgos ${ }^{2}$, Dimitrios G Goulis ${ }^{3}$ and Andromachi Vryonidou ${ }^{1}$ \\ 1Department of Endocrinology and Diabetes, Hellenic Red Cross Hospital, Athens, Greece \\ ${ }^{2}$ Department of Endocrinology and Diabetes, Sismanoglio-Amalia Fleming Hospital, Athens, Greece \\ ${ }^{3}$ Unit of Reproductive Endocrinology, First Department of Obstetrics and Gynecology, Medical School, \\ Aristotle University of Thessaloniki, Thessaloniki, Greece
}

Correspondence should be addressed to A Vryonidou Email mahi_vr@hotmail.com

\begin{abstract}
Objective: The aim of this study was to investigate the impact of adrenal hyperandrogenism on insulin resistance and lipid profile in women with polycystic ovary syndrome (PCOS).

Patients and methods: We studied 372 women with PCOS according to the NIH criteria. 232 age- and BMI-matched women served as controls in order to define adrenal hyperandrogenism (DHEA-S >95th percentile). Then, patients with PCOS were classified into two groups: with adrenal hyperandrogenism (PCOS-AH, $n=108)$ and without adrenal hyperandrogenism (PCOS-NAH, $n=264$ ). Anthropometric measurements were recorded. Fasting plasma glucose, insulin, lipid profile, sex hormone-binding globulin (SHBG) and androgen (TT, $\triangle 4 \mathrm{~A}, \mathrm{DHEA}-\mathrm{S}$ ) concentrations were assessed. Free androgen index (FAI) and homeostatic model assessment-insulin resistance (HOMA-IR) index were calculated. Results: Women with PCOS-AH were younger than PCOS-NAH $(P<0.001)$, but did not differ in the degree and type of obesity. No differences were found in HOMA-IR, total cholesterol, HDL-C, LDL-C and triglyceride concentrations (in all comparisons, $P>0.05)$. These metabolic parameters did not differ between the two groups even after correction for age. Women with PCOS-AH had lower SHBG $(29.2 \pm 13.8$ vs $32.4 \pm 11.8 \mathrm{nmol} / \mathrm{L}, P=0.025)$ and higher TT $(1.0 \pm 0.2 \mathrm{vs} 0.8 \pm 0.4 \mathrm{ng} / \mathrm{mL}, P=0.05)$ and $\triangle 4 \mathrm{~A}(3.9 \pm 1.2$ vs $3.4 \pm 1.0 \mathrm{ng} / \mathrm{mL}, P=0.007)$ concentrations, as well as FAl $(14.1 \pm 8.0 \mathrm{vs}$ $10.2 \pm 5.0, P<0.001)$. These results were confirmed by a multiple regression analysis model in which adrenal hyperandrogenism was negatively associated with age $(P<0.001)$ and SHBG concentrations $(P=0.02)$, but not with any metabolic parameter. Conclusions: Women with PCOS and adrenal hyperandrogenism do not exhibit any deterioration in insulin resistance and lipid profile despite the higher degree of total androgens.
\end{abstract} Key Words

Endocrine Connections (2017) 6, 601-606

\section{Introduction}

Polycystic ovary syndrome (PCOS) is characterized by chronic anovulation and hyperandrogenism (1). It is often accompanied by insulin resistance (2) and abnormal lipid profile $(3,4)$. Several studies have shown that insulin resistance is positively associated with the degree of hyperandrogenism $(5,6)$ and this was confirmed by a

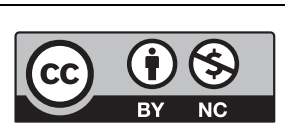


meta-analysis including 32 observational studies and 9556 women with PCOS in total. The higher the levels of androgens, the higher the degree of insulin resistance (7). Androgens are mainly of ovarian origin but in 30\% of the patients, they originate from the adrenal glands (8), the main representative being dehydroepiandrosterone sulfate (DHEA-S). The effect of this specific (mainly adrenal) androgen on the metabolic profile of women with PCOS has not yet been clarified.

The presence of adrenal androgen excess, expressed by elevated DHEA-S concentrations, has been postulated to contribute to a favorable metabolic $(9,10,11)$ and cardiovascular profile $(12,13)$ in women with PCOS, as well as in the general population $(14,15)$. However, scientific evidence is controversial, as other studies suggest a negative impact of adrenal hyperandrogenism on metabolic disturbances, including insulin resistance and hypertension in both PCOS (16) and in middle-aged individuals (17). Therefore, the exact influence of adrenal androgens on the metabolic aspects of PCOS remains inconclusive and requires further investigation.

The aim of this study was to investigate the impact of adrenal hyperandrogenism on insulin resistance and lipid profile in women with PCOS and its correlation with clinical and laboratory metabolic parameters.

\section{Subjects and methods}

\section{Patients and controls}

The study recruited 372 women with PCOS and 232 healthy controls. Patients with PCOS were selected from the outpatient clinics of two endocrine centers (Hellenic Red Cross Hospital and 'Sismanoglio-Amalia Fleming' Hospital). The enrolled control population consisted of medical or dietology students or hospitals' personnel. All participants provided written informed consent. Institutional Review Boards of the Hellenic Red Cross Hospital and 'Sismanoglio-Amalia Fleming' Hospital approved research procedures, while clinical investigations have been conducted according to the principles of the Declaration of Helsinki.

The National Institutes of Health (NIH) diagnostic criteria for PCOS were used, determined as the presence of less than eight menses per year and a free androgen index (FAI) greater than 5 and/or clinical hyperandrogenism (presence of acne and/or hirsutism) (18). Other causes of anovulation and hyperandrogenism were excluded. The control subjects had no history of menstrual irregularities and no clinical evidence of hyperandrogenism. Diabetes, hypertension, dyslipidemia and any other medical or psychiatric illness were excluded both in patients and controls. The use of oral contraceptives, anti-androgens or metformin was not reported for at least three months prior to the study.

Control group served as a means to define adrenal hyperandrogenism. The threshold of the 95th percentile of DHEA-S concentrations $(334 \mu \mathrm{g} / \mathrm{dL})$ in women with regular menstruation and no clinical hyperandrogenism was applied. According to this, patients with PCOS were divided into two groups: group A $(n=108)$ with adrenal hyperandrogenism (PCOS-AH) and group B $(n=264)$ without adrenal hyperandrogenism (PCOS-NAH).

\section{Study protocol}

Medical history of patients and controls was obtained and physical examination was performed by endocrinologists. Anthropometric measurements including weight, height and waist circumference (WC) were recorded. Body mass index (BMI) was calculated by the formula: (weight in $\mathrm{kg}$ )/ (height in $\mathrm{m}^{2}$ ). Clinical hyperandrogenism was assessed by the presence of acne and/or hirsutism (modified Ferriman-Gallwey score $>8$ ). Morning blood samples were drawn from all participants, after an overnight fast. Plasma glucose, insulin, total testosterone (TT), $\Delta 4$-androstenedione $(\Delta 4 \mathrm{~A}), \quad$ dehydroepiandrosterone sulfate (DHEA-S) and sex hormone-binding globulin (SHBG) were assessed in the early follicular phase of the menstrual cycle. Homeostatic model assessment-insulin resistance (HOMA-IR) index was calculated by using the mathematic model: HOMA-IR=glucose $\times$ insulin/405 (glucose in $\mathrm{mg} / \mathrm{dL}$ ) for the evaluation of insulin resistance (19). FAI was calculated by the formula: $\mathrm{FAI}=100 \times \mathrm{TT} \times 3.467 / \mathrm{SHBG}(\mathrm{TT}$ in $\mathrm{ng} / \mathrm{mL})$.

\section{Assays}

Assays were performed as previously described (20). Glucose concentrations were measured in plasma by an enzymatic, colorimetric method in a Cobas Integra/400/700/800 autoanalyzer (Roche Laboratory Systems). Insulin concentrations were measured in serum by an immunoradiometric assay (IRMA, DIASource Immunoassays S.A.) with a sensitivity of $1 \mu \mathrm{IU} / \mathrm{mL}$ and intra- and interassay coefficients of variation of $2.1 \%$ and $6.5 \%$, respectively. Lipid concentrations were measured by automatic biochemical analyzers. SHBG concentrations 
were measured in serum by an immunoradiometric assay (IRMA, Immunotech s.r.o.) with a sensitivity of $0.4 \mathrm{nmol} / \mathrm{L}$ and intra- and interassay coefficients of variation of 6.1 and $8.3 \%$, respectively. TT concentrations were measured by radioimmunoassay (RIA, Cisbio Bioassays) with a sensitivity of $0.086 \mathrm{ng} / \mathrm{mL}$ and intra- and interassay coefficients of variation of $6 \%$ and $8.5 \%$, respectively. $\triangle 4 \mathrm{~A}$ concentrations were measured by radioimmunoassay (RIA, DIASource Immunoassays S.A.) with a sensitivity of $0.03 \mathrm{ng} / \mathrm{mL}$ and intra- and interassay coefficients of variation of $4.5 \%$ and $9 \%$, respectively. DHEA-S concentrations were measured by radioimmunoassay (RIA, Immunotech s.r.o.) with a sensitivity of $2.64 \mu \mathrm{g} / \mathrm{dL}$ and intra- and interassay coefficients of variation of $4.93 \%$ and $9.32 \%$, respectively.

\section{Statistical analysis}

The study was powered to detect a 1.0 difference in HOMA-IR index, given a series of assumptions (HOMA-IR in PCOS-AH group: $4.0 \pm 2.5$; HOMA-IR in PCOS-NAH group: $3.0 \pm 2.5$; $\alpha$ error probability: $0.05, \beta$ error probability: 0.05 (power: 0.95), allocation ratio: $1 / 2$ (PCOS-AH/PCOS$\mathrm{NAH})$ ). According to these assumptions, 368 women had to be recruited (PCOS-AH: $n=123$; PCOS-NAH: $n=245$ ). Study power calculations were performed using the G*Power, version 3.1.9.2 (Heinrich Heine University, Dusseldorf, Germany).

Distribution of continuous parameters was tested by the Kolmogorov-Smirnov Test. Results are presented as absolute numbers (percentage) for categorical variables, while as mean \pm standard deviation (s.D.) for continuous variables. Differences in categorical variables between patients and controls were tested using $\chi^{2}$ test with Yates Correction. Differences in continuous variables between patients and controls were tested using the nonparametric Mann-Whitney $U$ test. Univariate Analysis of Variance was used to correct for age (age set as covariate). A multiple regression analysis model was used to evaluate the relationship between adrenal hyperandrogenism (DHEA-S/TT ratio being the dependent variable) and metabolic parameters. A $P$ value of $<0.05$ was considered statistically significant. All statistical analyses were performed using the Statistical Package for Social Sciences (SPSS 16.0, Inc).

\section{Results}

The metabolic and hormonal characteristics of two groups of women with PCOS are shown in Table 1 . Women with PCOS-AH were younger than PCOS-NAH $(P<0.001)$, but did not differ in the degree and type of obesity (assessed by BMI and WC, $P>0.05)$. No differences were found in HOMA-IR $(3.4 \pm 2.3$ vs $3.0 \pm 1.7)$, total cholesterol $(183 \pm 37$ vs $185 \pm 34 \mathrm{mg} / \mathrm{dL}), H D L-c(51.3 \pm 13.0 \mathrm{vs} 53.8 \pm 14.0 \mathrm{mg} / \mathrm{dL})$, LDL-c $(112 \pm 35$ vs $111 \pm 32 \mathrm{mg} / \mathrm{dL})$ and triglyceride $(83.1 \pm 35.0$ vs $87.3 \pm 49.0 \mathrm{mg} / \mathrm{dL})$ concentrations (in all comparisons, $P>0.05)$. Women with PCOS-AH had lower SHBG $(29.2 \pm 13.8$ vs $32.4 \pm 11.8 \mathrm{nmol} / \mathrm{L}, P=0.025)$ and

Table 1 Metabolic and hormonal characteristics of two groups of women with PCOS.

\begin{tabular}{lcc}
\hline Characteristics & PCOS-AH $(n=108)$ \\
\cline { 1 - 1 } Age (years) & $23.5 \pm 4.9$ \\
BMI $\left(\mathrm{kg} / \mathrm{m}^{2}\right)$ & $27.9 \pm 6.8$ \\
WC $(\mathrm{cm})$ & $88.2 \pm 12.8$ \\
Glu $(\mathrm{mg} / \mathrm{dL})$ & $83.1 \pm 7.7$ \\
Ins $(\mu \mathrm{lU} / \mathrm{mL})$ & $16.5 \pm 5.9$ \\
HOMA-IR & $3.4 \pm 2.3$ \\
Total cholesterol (mg/dL) & $183.0 \pm 37.0$ \\
HDL cholesterol $(\mathrm{mg} / \mathrm{dL})$ & $51.3 \pm 13.0$ \\
LDL cholesterol $(\mathrm{mg} / \mathrm{dL})$ & $112.0 \pm 35.0$ \\
Triglycerides $(\mathrm{mg} / \mathrm{dL})$ & $83.1 \pm 35.0$ \\
TT $(\mathrm{ng} / \mathrm{mL})$ & $1.0 \pm 0.2$ \\
DHEA-S $(\mu \mathrm{g} / \mathrm{dL})$ & $449.5 \pm 90.0$ \\
$\Delta 4 A(\mathrm{ng} / \mathrm{mL})$ & $3.9 \pm 1.2$ \\
SHBG $(\mathrm{nmol} / \mathrm{L})$ & $29.2 \pm 13.8$ \\
FAl & $14.1 \pm 8.0$ \\
\hline
\end{tabular}

\begin{tabular}{c}
\hline PCOS-NAH $(n=264)$ \\
\hline $26.3 \pm 6.3$ \\
$27.8 \pm 7.1$ \\
$87.0 \pm 12.0$ \\
$83.1 \pm 7.8$ \\
$14.6 \pm 4.1$ \\
$3.0 \pm 1.7$ \\
$185.0 \pm 34.0$ \\
$53.8 \pm 14.0$ \\
$111.0 \pm 32.0$ \\
$87.3 \pm 49.0$ \\
$0.8 \pm 0.4$ \\
$222.3 \pm 67.4$ \\
$3.4 \pm 1.0$ \\
$32.4 \pm 11.8$ \\
$10.2 \pm 5.0$
\end{tabular}

$\begin{array}{r}\hline \text { P value } \\ \hline<0.001 \\ 0.831 \\ 0.576 \\ 0.948 \\ 0.117 \\ 0.104 \\ 0.752 \\ 0.319 \\ 0.889 \\ 0.592 \\ 0.052 \\ <0.001 \\ 0.007 \\ 0.025 \\ <0.001 \\ \hline\end{array}$

$\boldsymbol{P}$ value after correction for age
-
0.322
0.239
0.718
0.133
0.113
0.795
0.388
0.807
0.541
0.196
$<0.001$
0.099
0.021
$<0.001$

$B M I$, body mass index; $\triangle 4 \mathrm{~A}, \triangle 4$-androstenedione; DHEA-S, dehydroepiandrostenedione sulfate; FAl, Free Androgen Index; HDL, high density lipoprotein; HOMA-IR, homeostatic model assessment-insulin resistance; LDL, low density lipoprotein; PCOS, polycystic ovary syndrome; PCOS-AH, polycystic ovary syndrome with adrenal hyperandrogenism; PCOS-NAH, polycystic ovary syndrome without adrenal hyperandrogenism; SHBG, sex hormone-binding globulin; TT, total testosterone; WC, waist circumference.

$\mathrm{FAI}=100 \times \mathrm{TT} \times 3.467 / \mathrm{SHBG}(\mathrm{TT}$ in $\mathrm{ng} / \mathrm{mL})$; HOMA-IR=glucose $\times$ insulin/405 (glucose in $\mathrm{mg} / \mathrm{dL})$.

http://www.endocrineconnections.org $\quad$ () 2017 The authors
$\begin{array}{ll}\text { DOI: } 10.1530 / \text { EC-17-0239 } & \text { Published by Bioscientifica Ltd }\end{array}$

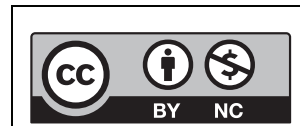

This work is licensed under a Creative Commons Attribution-NonCommercial 4.0 International License. 
Table 2 Multiple regression analysis model for women with PCOS and dependent variable the DHEA-S/TT ratio.

\begin{tabular}{|c|c|c|}
\hline Independent variables & Beta coefficient & $P$ value * \\
\hline Age & -0.320 & $<0.001$ \\
\hline BMI & 0.211 & 0.214 \\
\hline WC & -0.143 & 0.392 \\
\hline HOMA-IR & -0.178 & 0.075 \\
\hline Total cholesterol & -0.221 & 0.272 \\
\hline HDL cholesterol & 0.008 & 0.943 \\
\hline LDL cholesterol & 0.186 & 0.343 \\
\hline Triglycerides & -0.035 & 0.726 \\
\hline SHBG & -0.242 & 0.010 \\
\hline
\end{tabular}

*The same pattern was observed, when DHEA-S was set as the dependent variable.

BMI, body mass index; DHEA-S, dehydroepiandrostenedione-sulfate; HDL, high density lipoprotein; HOMA-IR, homeostatic model assessmentinsulin resistance; LDL, low density lipoprotein; PCOS, polycystic ovary syndrome; SHBG, sex hormone-binding globulin; TT, total testosterone; WC, waist circumference.

HOMA-IR, glucose $\times$ insulin/405 (glucose in $\mathrm{mg} / \mathrm{dL}$ ).

higher TT $(0.96 \pm 0.2$ vs $0.8 \pm 0.4 \mathrm{ng} / \mathrm{mL}, P=0.05)$ and $\Delta 4 \mathrm{~A}$ $(3.9 \pm 1.2$ vs $3.4 \pm 1.0 \mathrm{ng} / \mathrm{mL}, P=0.007)$ concentrations, as well as FAI $(14.1 \pm 8.0$ vs $10.2 \pm 5.0, P<0.001)$. As serum DHEA-S concentrations are heavily influenced by age in humans, we corrected the aforementioned comparisons for age and the $P$ value after this correction is presented in Table 1. The metabolic parameters did not differ between the two groups even after correction for age.

A multiple regression analysis followed, setting the DHEA-S/TT ratio as the dependent variable. We used this ratio in order for adrenal hyperandrogenism to be also adjusted for the ovarian hyperandrogenism. Age $(P<0.001)$ and SHBG concentrations $(P=0.02)$ were demonstrated to be negative predictors of adrenal hyperandrogenism. No association with any metabolic parameters was concluded from the multiple regression model (Table 2). The same pattern was observed, when DHEA-S was set as the dependent variable.

\section{Discussion}

This study provided evidence that women with PCOS and adrenal hyperandrogenism do not exhibit any additional deterioration of insulin resistance and lipid profile compared with women without adrenal hyperandrogenism.

Androgen excess, a cardinal feature of PCOS, has been experimentally incriminated as a potential developmental contributor to syndrome pathogenesis during fetal life $(20,21)$, but also as an independent aggravating factor of the metabolic disturbances during adolescent and adult life $(3,4,5,6,7,8,22)$. The co-existence of elevated adrenal and ovarian androgen production shown in the present study is in accordance with previous studies $(11,12,13)$ and may mirror the participation of the adrenal steroidogenesis to the total circulating steroid pool (23).

In the present study, when a cohort of women with PCOS was classified into two subgroups according to DHEA-S concentrations, no significant difference was observed between them in insulin resistance index and lipid profile, despite the higher concentrations of TT, $\Delta 4 \mathrm{~A}$ and FAI in the group with elevated DHEA-S concentrations. As serum DHEA-S concentrations are heavily influenced by age in humans, the aforementioned comparisons were corrected for age. Insulin resistance and lipid profile did not differ between the two groups even after this correction.

These data may demonstrate an independent effect of adrenal androgens that prevent further exacerbation of metabolic abnormalities in women with PCOS and high androgen concentrations. Previous studies have suggested that there is a beneficial impact of adrenal androgens on the metabolic phenotype in women with PCOS. Elevated DHEA-S concentrations have been inversely correlated with insulin resistance, estimated by HOMA-IR, in a PCOS cohort of over 350 women; this relationship was stronger than that of free testosterone or SHBG in the multivariate analysis (9). Furthermore, increased DHEA-S concentrations have been associated with a favorable lipid profile along with improved insulin sensitivity (assessed by quantitative insulin sensitivity check index - QUICKI) in a group of women with PCOS and hyperandrogenemia when compared to similar age and body weight patients with normal adrenal androgens (10). Another study including 318 untreated consecutive women with PCOS from Taiwan resulted again in inverse correlation of DHEA-S levels with the WC, waist-to-hip ratio, BMI, insulin resistance, $\mathrm{LDL}$ and triglycerides levels (11). Of great importance, DHEA-S concentrations were shown to be inversely correlated with the carotid intimal media thickness in earlier studies, suggesting not only favorable metabolic effects but also cardioprotective ones for endogenous DHEA-S in women with PCOS $(12,13)$.

The underlying pathogenic mechanisms of these findings remain unclear, but may reflect a direct effect of DHEA-S on insulin (24) and lipid metabolism (25). Dehydroepiandrosterone (DHEA) concentrations have been positively correlated to insulin binding activity 
(24), suggesting a direct impact on insulin physiology. On the other hand, insulin was experimentally reported to enhance DHEA-S production through a direct effect on the adrenal gland itself (26). Interestingly, in the present study, a negative correlation between adrenal hyperandrogenism and SHBG concentrations was demonstrated. Given that low SHBG concentrations are associated with insulin resistance (27), the elevated DHEA-S concentrations could reflect an adaptive mechanism to this metabolic disturbance, implying further complexity in the interplay between hormones and energy homeostasis (28). Furthermore, the negative correlation between adrenal androgens with age demonstrated in the present study is in accordance with previous findings of an age-related reduction in DHEA-S concentrations in the general population (29) as well as in women with PCOS (30).

The strengths of the study include the use of NIH criteria, as well as the well-defined population of Caucasian women only with similar socio-economic status. A limitation of the study could be the sample size from an epidemiological point of view. However, it is one of the largest in this research topic.

In conclusion, this study provided evidence that the presence of adrenal hyperandrogenism, defined by elevations in DHEA-S concentrations, may constitute a factor that prevents further deterioration of metabolic profile (insulin resistance, lipid abnormalities) in women with PCOS. It remains to be clarified whether higher DHEA-S concentrations are an adaptive mechanism to insulin resistance or they exert a protective role on the metabolic profile of women with PCOS.

\section{Declaration of interest}

The authors declare that there is no conflict of interest that could be perceived as prejudicing the impartiality of the research reported.

\section{Funding}

This research did not receive any specific grant from any funding agency in the public, commercial or not-for-profit sector.

\section{References}

1 Conway G, Dewailly D, Diamanti-Kandarakis E, Escobar-Morreale HF, Franks S, Gambineri A, Kelestimur F, Macut D, Micic D, Pasquali R, et al. The polycystic ovary syndrome: a position statement from the European Society of Endocrinology. European Journal of Endocrinology 2014171 P1-P29. (doi:10.1530/EJE-14-0253)

2 Diamanti-Kandarakis E \& Dunaif A. Insulin resistance and the polycystic ovary syndrome revisited: an update on mechanisms and implications. Endocrine Reviews 201233 981-1030. (doi:10.1210/ er.2011-1034)
3 Wild RA. Dyslipidemia in PCOS. Steroids 201277 295-299. (doi:10.1016/j.steroids.2011.12.002)

4 Vryonidou A, Paschou SA, Muscogiuri G, Orio F \& Goulis DG. Mechanisms in endocrinology: metabolic syndrome through the female life cycle. European Journal of Endocrinology 2015173 R153-R163. (doi:10.1530/EJE-15-0275)

5 Goverde AJ, van Koert AJ, Eijkemans MJ, Knauff EA, Westerveld HE, Fauser BC \& Broekmans FJ. Indicators for metabolic disturbances in anovulatory women with polycystic ovary syndrome diagnosed according to the Rotterdam consensus criteria. Human Reproduction 200924 710-717. (doi:10.1093/humrep/den433)

6 Castelo-Branco C, Steinvarcel F, Osorio A, Ros C \& Balasch J. Atherogenic metabolic profile in PCOS patients: role of obesity and hyperandrogenism. Gynecological Endocrinology 201026 736-742. (doi:10.3109/09513590.2010.481025)

7 Yang R, Yang S, Li R, Liu P, Qiao J \& Zhang Y. Effects of hyperandrogenism on metabolic abnormalities in patients with polycystic ovary syndrome: a meta-analysis. Reproductive Biology and Endocrinology 201614 67. (doi:10.1186/s12958-016-0203-8)

8 Yildiz BO \& Azziz R. The adrenal and polycystic ovary syndrome. Reviews in Endocrine and Metabolic Disorders 20078 331-342. (doi:10.1007/s11154-007-9054-0)

9 Brennan K, Huang A \& Azziz R. Dehydroepiandrosterone sulfate and insulin resistance in patients with polycystic ovary syndrome. Fertility and Sterility 200991 1848-1852. (doi:10.1016/j. fertnstert.2008.02.101)

10 Carmina E \& Lobo RA. Prevalence and metabolic characteristics of adrenal androgen excess in hyperandrogenic women with different phenotypes. Journal of Endocrinological Investigation 200730 111-116. (doi:10.1007/BF03347408)

11 Chen MJ, Chen CD, Yang JH, Chen CL, Ho HN, Yang WS \& Yang YS. High serum dehydroepiandrosterone sulfate is associated with phenotypic acne and a reduced risk of abdominal obesity in women with polycystic ovary syndrome. Human Reproduction 201126 227-234. (doi:10.1093/humrep/deq308)

12 Vryonidou A, Papatheodorou A, Tavridou A, Terzi T, Loi V, Vatalas IA, Batakis N, Phenekos C \& Dionyssiou-Asteriou A. Association of hyperandrogenemic and metabolic phenotype with carotid intimamedia thickness in young women with polycystic ovary syndrome. Journal of Clinical Endocrinology and Metabolism 200590 2740-2746. (doi:10.1210/jc.2004-2363)

13 Meyer C, McGrath BP, Cameron J, Kotsopoulos D \& Teede HJ. Vascular dysfunction and metabolic parameters in polycystic ovary syndrome. Journal of Clinical Endocrinology and Metabolism 200590 4630-4635. (doi:10.1210/jc.2004-1487)

14 Brahimaj A, Muka T, Kavousi M, Laven JS, Dehghan A \& Franco OH. Serum dehydroepiandrosterone levels are associated with lower risk of type 2 diabetes: the Rotterdam Study. Diabetologia 201760 98-106. (doi:10.1007/s00125-016-4136-8)

15 Mannic T, Viguie J \& Rossier MF. In vivo and in vitro evidences of dehydroepiandrosterone protective role on the cardiovascular system. International Journal of Endocrinology and Metabolism 201513 e24660.

16 Alpañés M, Luque-Ramírez M, Martínez-García MÁ, FernándezDurán E, Álvarez-Blasco F \& Escobar-Morreale HF. Influence of adrenal hyperandrogenism on the clinical and metabolic phenotype of women with polycystic ovary syndrome. Fertility and Sterility 2015 103 795-801.

17 Schunkert H, Hense HW, Andus T, Riegger GA \& Straub RH. Relation between dehydroepiandrosterone sulfate and blood pressure levels in a population-based sample. American Journal of Hypertension 199912 1140-1143.

18 Zawadski JK \& Dunaif A. Diagnostic criteria for polycystic ovary syndrome: towards a rational approach. In Polycystic Ovary Syndrome, pp 377-384. Eds A Dunaif, JR Givens, FP Haseltine \& GR Merriam. Boston, MA, USA: Blackwell Scientific Publications, 1992. 
19 Matthews DR, Hosker JP, Rudenski AS, Naylor BA, Treacher DF \& Turner RC. Homeostasis model assessment: insulin resistance and betacell function from fasting plasma glucose and insulin concentrations in man. Diabetologia 198528 412-419. (doi:10.1007/BF00280883)

20 Paschou SA, Ioannidis D, Vassilatou E, Mizamtsidi M, Panagou M, Lilis D, Tzavara I \& Vryonidou A. Birth weight and polycystic ovary syndrome in adult life: is there a causal link? PLOS ONE $2015 \mathbf{1 0}$ e0122050. (doi:10.1371/journal.pone.0122050)

21 Franks S, McCarthy MI \& Hardy K. Development of polycystic ovary syndrome: involvement of genetic and environmental factors. International Journal of Andrology 200629 278-285. (doi:10.1111/ j.1365-2605.2005.00623.x)

22 Huang R, Zheng J, Li S, Tao T, Ma J \& Liu W. Characteristics and contributions of hyperandrogenism to insulin resistance and other metabolic profiles in polycystic ovary syndrome. Acta Obstetricia et Gynecologica Scandinavica 201594 494-500. (doi:10.1111/aogs.12612)

23 Goodarzi MO, Carmina E \& Azziz R. DHEA, DHEAS and PCOS. Journal of Steroid Biochemistry and Molecular Biology 2015145 213-225. (doi:10.1016/j.jsbmb.2014.06.003)

24 Buffington CK, Given JR \& Kiabchi AE. Opposing actions of dehydroepiandrosterone and testosterone on insulin sensitivity. In vivo and in vitro studies of hyperandrogenic females. Diabetes 1991 40 693-700. (doi:10.2337/diab.40.6.693)
25 Lea-Currie YR, Wen P \& McIntosh MK. Dehydroepiandrosteronesulfate (DHEAS) reduces adipocyte hyperplasia associated with feeding rats a high-fat diet. International Journal of Obesity and Related Metabolic Disorders 199721 1058-1064. (doi:10.1038/sj.ijo.0800516)

26 Hines GA, Smith ER \& Azziz R. Influence of insulin and testosterone on adrenocortical steroidogenesis in vitro: preliminary studies. Fertility and Sterility 200176 730-735. (doi:10.1016/S00150282(01)02014-3)

27 Haffner SM. Sex hormone-binding protein, hyperinsulinemia, insulin resistance and noninsulin-dependent diabetes. Hormone Research 199645 233-237. (doi:10.1159/000184794)

28 Triantafyllou GA, Paschou SA \& Mantzoros CS. Leptin and hormones: energy homeostasis. Endocrinology Metabolism Clinics of North America 201645 633-645. (doi:10.1016/i.ecl.2016.04.012)

29 Sulcová J, Hill M, Hampl R \& Stárka L. Age and sex related differences in serum levels of unconjugated dehydroepiandrosterone and its sulphate in normal subjects. Journal of Endocrinology 1997 $15457-62$

30 Rashidi BH, Gorginzadeh M, Aalipour S \& Sills ES. Age related endocrine patterns observed in polycystic ovary syndrome patients vs ovulatory controls: descriptive data from a university based infertility center. Archives of Endocrinology and Metabolism 201660 486-491. (doi:10.1590/2359-3997000000215)

Received in final form $\mathbf{8}$ September 2017

Accepted 14 September 2017

Accepted Preprint published online 14 September 2017 http://www.endocrineconnections.org DOI: 10.1530/EC-17-0239 (c) 2017 The authors Published by Bioscientifica Ltd

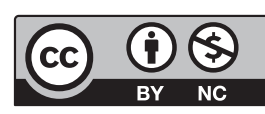

This work is licensed under a Creative Commons Attribution-NonCommercial 4.0 International License. 